\title{
Effect of Side Wind on the Directional Stability and Aerodynamics of a Hybrid Buoyant Aircraft
}

\author{
Anwar U Haque ${ }^{1}$, Waqar Asrar ${ }^{1}$, Ashraf A Omar ${ }^{2}$, Erwin Sulaeman ${ }^{1}$ and Mohamed J.S Ali ${ }^{1}$ \\ 1 International Islamic University Malaysia (IIUM), 50728 Kuala Lumpur, Malaysia \\ ${ }^{2}$ University of Tripoli (UOT), P.O. Box 13154 Tripoli-Libya
}

\begin{abstract}
Directional stability characteristics explain the capabilities of a hybrid buoyant aircraft's performance against the side wind, which induces flow separation that is chaotic in nature and may lead to oscillations of the aerodynamic surfaces. A numerical study is carried out to estimate the effect of side wind. The boundary conditions for the computational domain are set to velocity inlet and pressure outlet. Due to the incompressible flow at the cruise velocity, the density is taken to be constant. For these steady state simulations, the time is discretized in first order implicit and the SIMPLE scheme is employed for pressure velocity coupling alongwith $k$ - $\omega S S T$ model. Based on the results obtained so far, it is concluded that voluminous hybrid lifting fuselage is the major cause of directional.
\end{abstract}

\section{Introduction}

It is known historically that there are basically two kinds of aircraft, one is conventional aircraft and other one is unconventional. Conventional as well as unconventional aircraft are accelerated either by using forward thrust or utilizing the VTOL technology [1] . However, there is another type of aircraft, known as hybrid buoyant (HB) aircraft [2]. Such aircraft takeoff and land similar to any other aircraft but utilizes aerostatic lift which is usually filled in hulls of conventional shapes [3]. Moreover, the aerodynamic contour of the hull of HB aircraft can be designed to contribute some percentage of lift for acceleration as well as for take-off flight [4]. Different tasks performed by such vehicles include transportation of agricultural goods, transportations of tourists and spraying of agricultural chemicals etc [5]. For all these purposes, speed and altitude of such vehicle is less as compared with the conventional aircraft and for which the directional stability of the vehicle is mandatory.

With the fossil fuel deposits getting depleted fast and the noise suppression becoming an important area, novel ideas are being explored relentlessly in the aviation industry. For an aircraft, in order to avoid stall during the take-off and landing in particular, deployment of high lift devices is a common practice in vogue [6]. However, it will increase the drag coefficient and hence more fuel of aircraft will be burned [7]. Presently, there is an urge in the industry to tackle these important issues by using novel flow control techniques such as synthetic jets [8]. As partial takeoff mass is balanced by aerostatic lift, therefore, $H B$ aircraft solve this problem to some extent [9]. Designer of such an aircraft needs to be well versed in directional stability analysis. Key information of the systems used in the estimation of directional stability characteristics include airframes/geometric definitions and the flow conditions for the specified flight segment [10]. Low fidelity tools can be used for the said purpose. Suh tools like Aircraft DATCOM is part of other analysis software like CEASIOM. But the contribution of lift produced by the fuselage cannot be estimated by this analytical tool. Hence, its utilization for $H B$ aircraft's directional stability will be inadequate. In the present work, for the fixed locations of the stability surfaces and $C G$ location at $15.2 \mathrm{~m}$, contribution of individual components of the $H B$ aircraft towards directional stability analysis is estimated by using component buildup method in $C F D$. Though the aircraft is stable for the defined conditions, but it can be improved in future by employing multiple dorsal fins.

\section{Methodology}

The methodology adopted for the present computational work is the same as used for any numerical simulation. All sides of the computational domain are defined as velocity inlet, except that the back face is taken as pressure outlet. Commercial CFD software, FLUENT [11] is used with all the solution methods set to the default. This include gradient-least squares cell based discretization with first order upwind scheme for viscous flow analysis [11]. Boundary conditions were defined for individual components to estimate there contribution towards directional stability No slip condition is used for all components of HB aircraft. Special emphasis was given for the grid generation as well for controlling the $C F L$ number during initial runs. All the parameters were non-dimensionalized $S_{\text {wing. }}$ 


\section{Geometry description and grid generation}

The model generated using "CATIA" is used for grid generation in "ICEM-CFX" software. Special attention is paid for the grid clustering in areas where the aerodynamic (lifting as well as non-lifting) surfaces are attached with the voluminous fuselage.

\subsection{Geometry description}

The dimensions of major geometric parameters have already been discussed in reference [12]. It has a wing span of $20 \mathrm{~m}$ and uses canards to decrease the pressure height. About 32 meter long fuselage also produces aerodynamic lift along with the aerostatic lift due to the lifting gas filled in it, Fig. 1. Its wing contributes about $50 \%$ of the total lift requirement in cruise and is located at rear position of the neutral point. In such design, structural weight constraint and added mass affects limit the use of elongated vertical tails of the H-tail assembly.

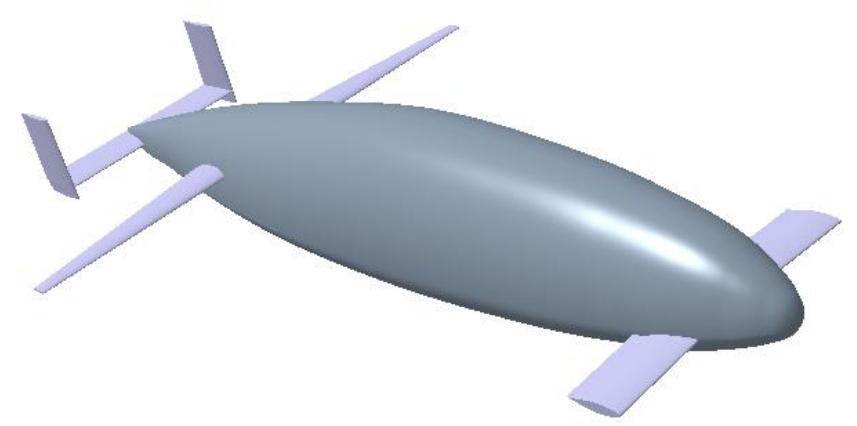

Figure 1. Schematic Views of HB aircraft

\subsection{Grid Generation}

The ICEM software is used to generate the $C$-O type three-dimensional grid and special attention has been paid for the grid refinement at the junction and edges of the aerodynamic surfaces, Fig. 2. Specially grid is refined at the nose of hull, Wing -HLF Junction and VentralVertical Tail Junction.

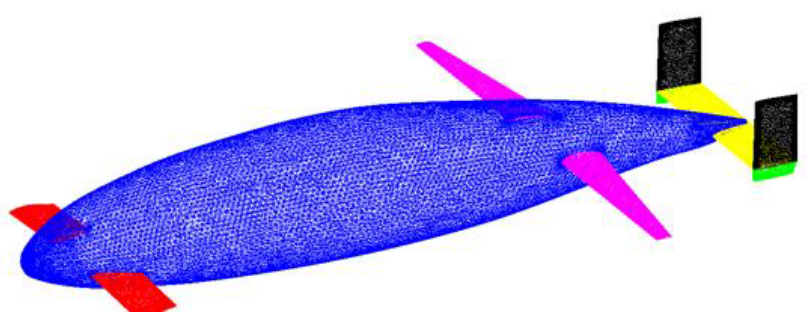

Figure 2. Surface Grid

\section{Numerical analysis}

$C F D$ is a well-known technique that enables to study the flow characteristics of a body immersed in a fluid [13].
$C F D$ as a computational technology is incorporated in the conceptual design software [14]. Such s software can estimate the forces acting on the body by solving complete set of flow with Euler assumption [14]. But the element of turbulence modeling is perhaps missing which plays a dominant role in flow simulations and it requires large computer run time and computer memory. At the same time, the accuracy of the numerical results depends largely on the quality of the fine mesh. Wall adaptation can save the computational cost as not very fine grid is required to resolve the boundary layer features, specially at the mating junction of the fuselage and the aerodynamic surfaces. In this section we provide a brief description of analytical methods and procedures followed for estimation of stability Equations should be centred and should be numbered with the number on the right-hand side.

\section{Results and discussion}

It is well known that a very fine mesh size is required for CFD work that resolves the fully turbulent region, including the buffer layer and viscous sub layer. But the numerical cost is quite high specially for the CFD analysis of the complete configuration of HB aircraft. A wall function approach resolves this issue to some extent, without incurring added mesh generation and computational time. In the present work, non-equilibrium wall function approach is employed for which the initial mesh should have wall $\mathrm{y}+$ in the range of 30 to 60 [18]. $\mathrm{y}+$ distribution on the canard and the wing of the configuration under discussion are shown in Fig. 3.

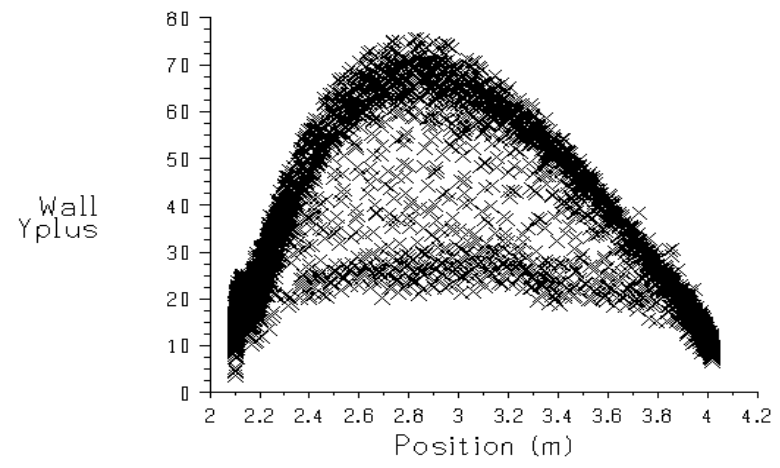

(a) Canard

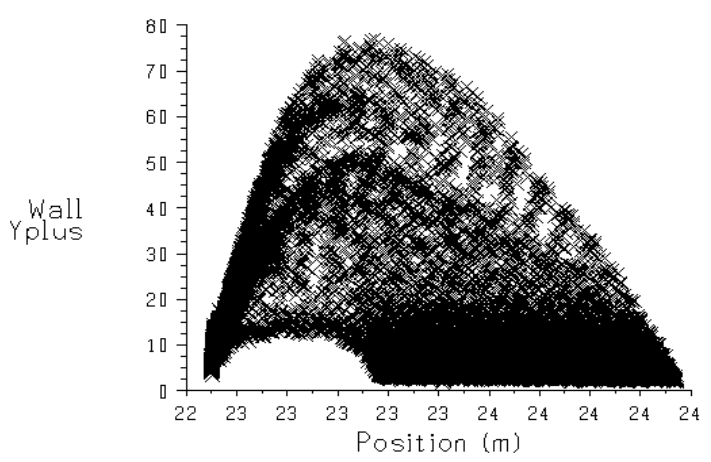

(b) Wing

Figure 3. y-plus distribution over the surface 
The steady solutions are run on an Intel hex-core dual processor, $3.33 \mathrm{GHz}$ system with $32 \mathrm{~GB}$ RAM. Sample results alongwith the mesh details are shown in Tab. 1:

Table 1. Sample results at $\alpha$ and $\beta=0^{\circ}$

\begin{tabular}{|c|c|c|c|c|c|}
\hline Type & Faces & Nodes & $C_{L}$ & $C_{D}$ & $C_{N}$ \\
\hline $\begin{array}{c}\text { unstruct- } \\
\text { ured }\end{array}$ & 110600033 & 1445849 & 0.66 & 0.138 & -0.0003 \\
\hline
\end{tabular}

The convergence criteria for all the simulations are taken as $10-5$ for continuity and energy equation. However, for $\mathrm{x}, \mathrm{y}, \mathrm{z}$ velocities and others quantities, it is 10-6. In the simulation work, the cruise velocity is 28.69 $\mathrm{m} / \mathrm{s}$ and the Reynolds number (based on the mean aerodynamic chord) is $2.27 \times 105$.

\subsection{Aerodynamics}

For the estimation of the aerodynamic coefficients, the axis system is defined such that we have $x$-axis along the body, $y$-axis in upward direction and $z$-axis along the span (left wing). We define the components of the velocity in Tab. 2 to simulate $\beta$ such that $C_{L}$ coordinate is upward and its angle is $90^{\circ}$ to the body axis, thus it is not affected by sideslip angle. It can be observed that a negative sign appears for the defining vector of $C_{D}$ because the vertical component of the velocity will produce force opposite to the force generated by the axial velocity.

Table 2. Cordinates of the force vector for defining $\beta$

\begin{tabular}{|c|c|c|}
\hline Orientation & $C_{L}(x, y, z)$ & $C_{D}(x, y, z)$ \\
\hline$+\beta$ & $(0,1,0)$ & $(\cos \beta, 0,-\sin \beta)$ \\
\hline
\end{tabular}

Initial CFD results have shown that the rear part of the fuselage is contributing more towards creating the pressure difference between its windward and leeward side, Fig. 4. The camber of the fuselage generates the lift which is in addition to the lift produced by the symmetric vortices at high $\alpha$ [12]. With increase in $\beta$, except the wing, the $C_{D}$ count remains almost constant till $\beta>4 \mathrm{o}$, but this behavior of the wing is not observed in $C_{L}$, Fig. 5(a) and Fig 5(b). The decrease in lift and increased drag will collectively result in the overall lower aerodynamic efficiency after $\beta>50$ and hence the fuel consumption will increase during yaw and will decrease the overall flight range. After $\beta>110$, a drastic decrease in $C_{D}$ as well as in $C_{L}$ is observed which drop to almost zero for $\beta>15$ o. It is important to highlight that the upwash and downwash of wing alone and wing-fuselage will totally be different. Hence any sort of CFD can give an answer but it is difficult to separate out the portion of the yawing moment caused by the fuselage, since the wing and fuselage affect each other so much. Moreover, one cannot generate the CFD results of hybrid lifting fuselage (HLF) tests with upwash and downwash for validation with analytical approach like Munk-Multhop Method for estimation of yawing moment due to the fuselage, as there is no wing attached to it.

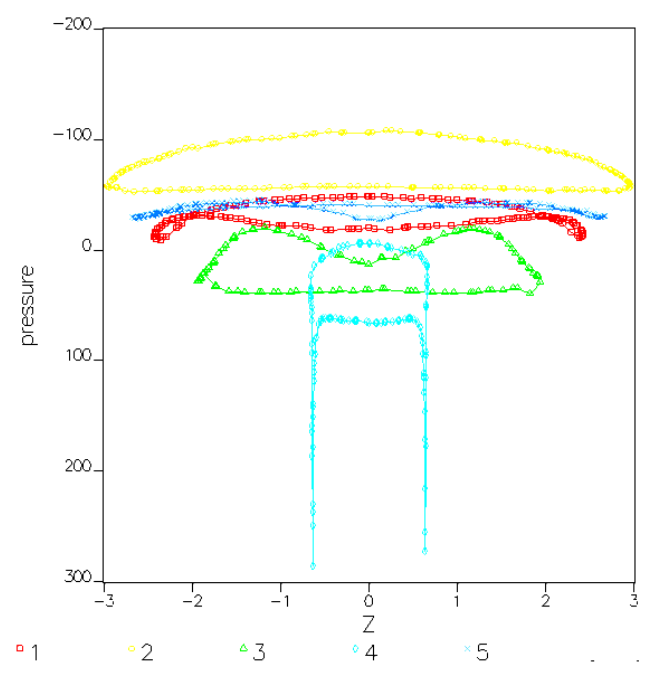

(b) Pressure distribution for the defined sections

Figure 4. Surface pressure distribution $(\mathrm{Pa})$ on defined sections of HLF at $\beta=0$ o

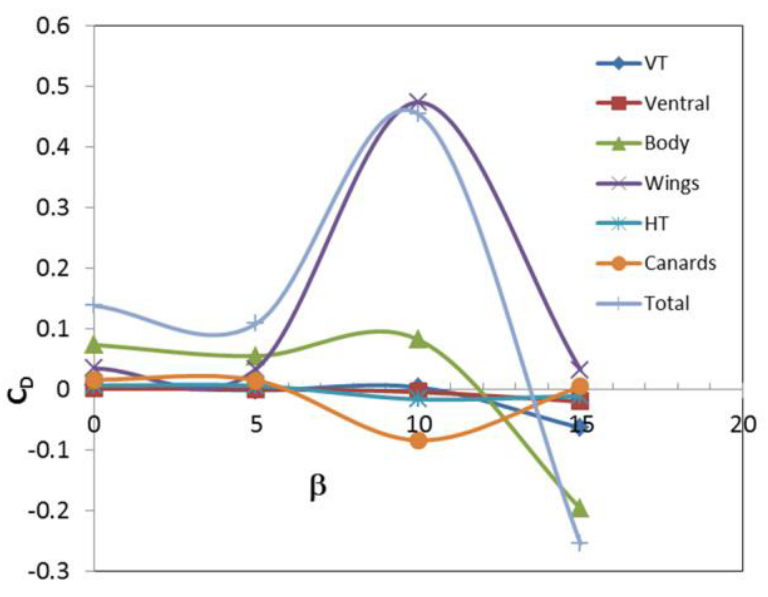

(a) $C_{D}$ vs $\beta$

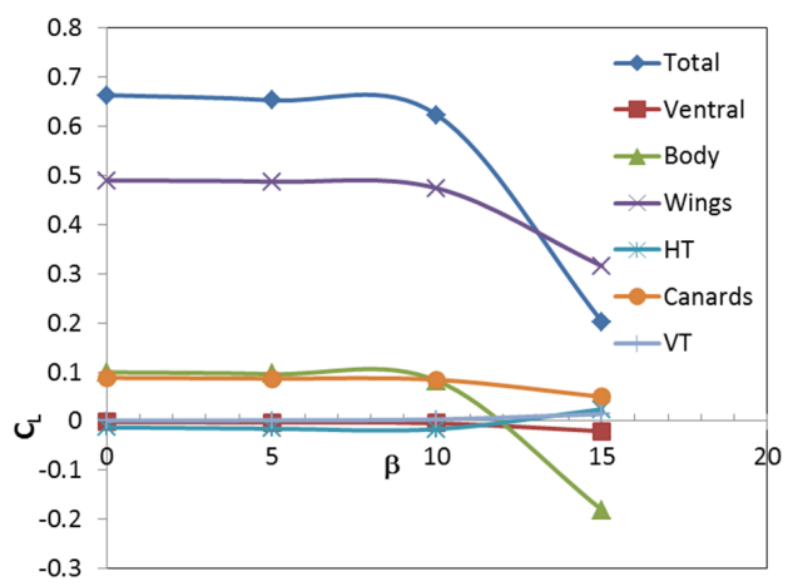

(b) $C_{L}$ vs $\beta$

Figure 5. Variation in aerodynamic coefficients $\beta$ 
Wind tunnel testing in the presence and absence of the wing for upwash and downwash of complete body will be the best representative of the actual scenario. Infact, HLF's yawing moment is mostly caused by the upwash and downwash of the wing, applied to the fuselage, and is almost always positive (destabilizing).

\subsection{Directional Stability}

When the airplane is subjected to positive sideslip angle $\beta$, static directional stability is evident if a positive yawing moment coefficient results [19]. Therefore if the relative wind comes from the right, a yawing moment to the right should be created which tends to weather cock the HB aircraft and return the nose into the wind. Therefore for the airplane to be directionally stable, the slope of $C_{n}-\beta$ curve should be positive [20]. The amount of stability is dependent upon the amount of slope. If the slope is zero, then the airplane is neutrally stable. In order to get a first order estimation of the said slope, first the contribution of each component of HB aircraft has been evaluated by using the Eq. (1) followed by plotting the trend line of 4 th order polynomial on the total yawing moment coefficient, non-dimensionalized by the total wing span of $20 \mathrm{~m}$.

$$
C_{n_{\beta}}=C_{n_{\beta_{w}}}+C_{n_{\beta_{f u s}}}+C_{n_{\beta H T}}+C_{n_{\beta_{V T}}}+C_{n_{\beta_{c}}}
$$

Graph of Fig. 6 shows the contribution of individual components towards yawing moment, out of which the main contributor towards directional stability is vertical tail. When the HB aircraft experiences $\beta$, the local angle of attack of the vertical tail changes and thus changes its lift. Contribution of wing towards directional stability is very small. The contribution of the fuselage is of primary importance since it furnishes the greatest destabilizing influence. The ventral's contribution is quite small but stabilizing when compared with that of the wing. For $\beta=0 \mathrm{o},{ }^{C_{n}}$ of this configuration is equal to -0.0004 .

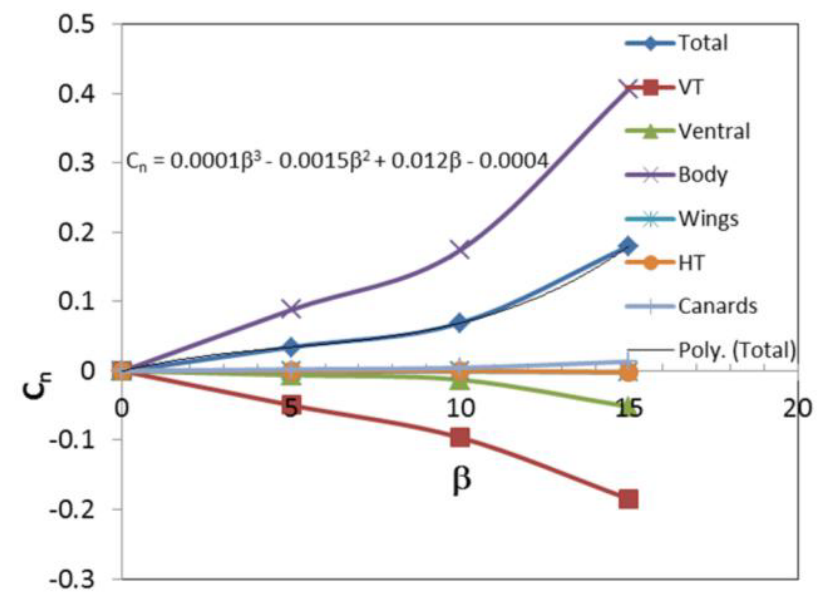

Figure 6. Variation in $C_{n}$ vs $\beta$
$C_{n}$ (per degree) obtained from Fig. 6 is equal to 0.012 . This variable is positive, which shows that the aircraft is stable This value is perhaps double of the value obtained earlier by using XFLR [12]. This is due to the under prediction in the lift coefficients by XFLR and its limitation for not capturing the pre stall characteristics, where mild flow separation of the boundary layer can cause additional drag and loss of lift. Its value can be increased by using additional dorsal fins like that on the shark fish. Streamline plots for $\beta=00$ has shown that flow remains attached with the aerodynamic surfaces, Fig. 7. Moreover, the pressure increases when $\beta=100$, where the skin friction lines show an asymmetric pattern, causing one wing to generate more lift in comparison with the other wing.

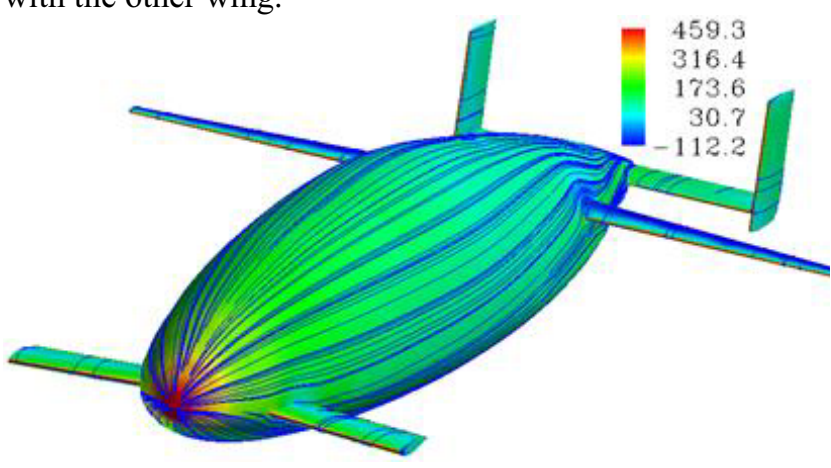

(a) $\beta=0^{0}$

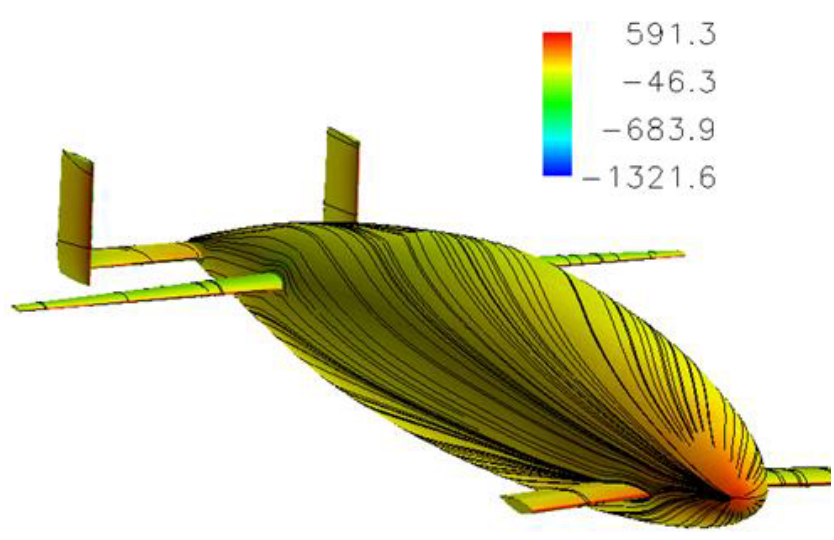

(b) $\beta=10^{0}$

Figure 7. Streamline plot with contours of

total pressure $(\mathrm{Pa})$

\subsection{Future Work}

In this paper, we have discussed only the directional stability characteristics for the cruise segment. However, after validation of the computational results from the wind tunnel testing, this work can be extended in future for other flight segments as well, like takeoff segment. Moreover, in order to capture the flow physics of vortical flow on the HLF at moderate angle of attack, there will be requirement for fine mesh in leeward side of HLF. 
The undesirable instability contribution by the voluminous fuselage requires large size empennage to make such an aircraft directionally stable, which in return makes the tail quite heavy in weight. Similar to the multiple fins on the elongated bodies of shark, additional dorsal fins at different aft location from the CG is one of the prospective solutions, the application of which for $\mathrm{HB}$ aircraft is yet to be explored [10]. On the other side, with a slight yaw, dorsal fin causes a lot of drag and it can produce a restoring yawing moment but not that enough such as a rudder produces. Moreover, a dorsal fin can be utilized to house an external antenna, commonly used for the communication and navigation systems [20]-[21].

As a nutshell, all the said points are active and extremely challenging research areas of fundamental importance. Any fruitful research in this area will not only augment to a better scientific understanding, but will also serve towards attaining the future breakthroughs in preliminary design of HB aircraft.

\section{Conclusion}

In this research article, the $\mathrm{HB}$ aircraft is found to be directionally stable as the sign of the slope of $C_{n \beta}$ is positive. For the specified range of $\beta$, HLF and canards produce same order of lift. At low angle of attack, HLF contributions towards drag are more than that of the wing. With increase in $\beta$, trends of $C_{n \beta}$ of all the components of HB aircraft are consistent. Better and realistic results can only be produced if the grid is developed such that the condition of $\mathrm{y}+$ equal to one is met, which will definitely require advanced computing power.

\section{References}

1. A. S. Gohardani, "A synergistic glance at the prospects of distributed propulsion technology and the electric aircraft concept for future unmanned air vehicles and commercial/military aviation," Prog. Aerosp. Sci., 57, pp. 25-70, Feb. (2013).

2. A. U. Haque, W. Asrar, A. A. Omar, E. Sulaeman, and J. M. Ali, "Stability and Takeoff Ground Roll Issues of Hybrid Buoyant Aircraft," In Applied Mechanics and Materials, 660, pp. 503-507. (2014).

3. A. U. Haque, W. Asrar, A. A. Omar, E. Sulaeman, and J. M. Ali, "A Novel Design of a Hybrid Buoyant Aircraft- A Potential Greener Solution for Inter Connectivity of Malaysian Islands," AER0 15, 19-21 April, Monterial Canada. (2015).

4. A. U. Haque, W. Asrar, A. A. Omar, E. Sulaeman, and J. M. Ali, "Comparison of Digital DATCOM and Wind Tunnel Data of a Winged Hybrid Airship's Generic Model," Appl. Mech. Mater. Trans Tech Publ. Switz., vol. 629, pp. 36-41, (2014).

5. A. U. Haque, W. Asrar, A. a. Omar, E. Sulaeman, and J. M. Ali, "Conceptual Design of a Winged Hybrid Airship," $21^{\text {st }}$ AIAA Light. Syst. Technol. Conf., pp. AIAA-2014-2710, (2014).
6. A. Robert Majka, "Take-off aided by magnetic levitation technology," Aircr. Eng. Aerosp. Technol., 85 6, pp. 435-442, (2013).

7. A. S. Gohardani, G. Doulgeris, R. Singh, and A. S. G. $\tilde{\mathrm{A}}$, "Challenges of future aircraft propulsion: A review of distributed propulsion technology and its potential application for the all electric commercial aircraft," Prog. Aerosp. Sci., 47 5, pp. 369-391, Jul. (2011).

8. X. Fei and Y. Zhengyin, "Drag Reduction for an Airship with Proper Arrangement of Propellers," Chinese J. Aeronaut., 22 6, pp. 575-582, Dec. (2009).

9. A. U. Haque, W. Asrar, A. A. Omar, E. Sulaeman, and J. M. Ali, "Pugh Analysis for Configuration Selection of a Hybrid Buoyant Aircraft," No. 201501-2446. SAE Technical Paper, (2015).

10. J. D. Anderson, Aircraft Performance and Design. Boston: McGraw-Hill, (1999).

11. Fluent Manual," "Known Limitations in ANSYS FLUENT 14. (2013).

12. A. U. Haque, W. Asrar, A. a. Omar, E. Sulaeman, and J. M. Ali, "Power-off static stability analysis of a clean configuration of a hybrid buoyant aircraft", 7th Ankara International aerospace conference, METU, Ankara Turkey. 11-13 September (2015).

13. A. Cenko, "One CFD calculation to end point flight testing," Aeronaut. J., vol. 110, no. 1109, pp. 439446, (2006).

14. R.Von Kaenel, A. Rizzi, , J.,Oppelstrup, Goetzendorf-Grabowski, CEASIOM: simulating stability \& control with CFD/CSM in aircraft conceptual design. In 26th International Congress of the Aeronautical Sciences, ICAS. (2008)

15. H. Lomax, T. Pulliam, D. Zingg, and T. Kowalewski, "Fundamentals of Computational Fluid Dynamics," Applied Mechanics Reviews, 55 4. p. B61, (2002).

16. Anwar-ul-Haque, J. Khawar, and S. Raza Ch, "Influence of Turbulence Modeling In Capturing Separated Flow Over Delta Wing At Subsonic Speed," Eng. Appl. Comput. Fluid Mech., 2 3, pp. 252-263, 2008.

17. K. Pettersson, CFD Methods for Predicting Aircraft Scaling Effects. (2008).

18. H. Zaïdi, S. Fohanno, R. Taïar, and G. Polidori, "Turbulence model choice for the calculation of drag forces when using the CFD method," J. Biomech., 43 3, pp. 405-411, (2010).

19. M. V. Cook, Flight dynamics principles. 2007.

20. D. Raymer, Aircraft Design: A Conceptual Approach, Fifth Edit. Washington, DC: American Institute of Aeronautics and Astronautics, Inc., (2012).

21. C. Droooping, D. Antenna, and M. Amin, "Wireless Communication \& Radar. (1994).

22. M. M. Karbassian, "Phase-Modulations Analyses in Coherent Homodyne Optical CDMA Network Using a Novel Prime Code Family," Engineering, 1, pp. 48, (2007). 